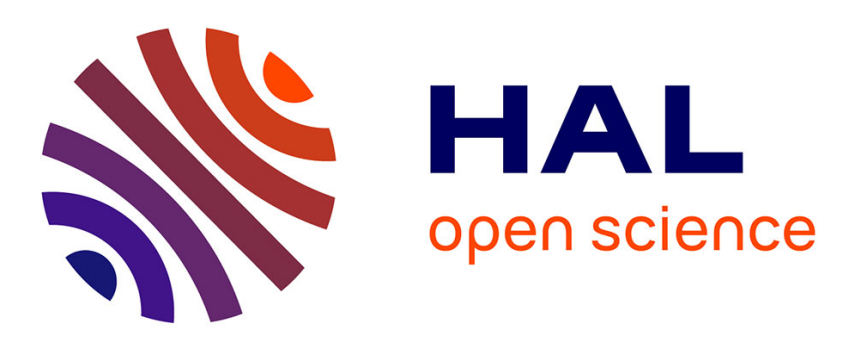

\title{
Démonstratif et déixis discursive: analyse comparée d'un corpus écrit de français médiéval et d'un corpus oral de français contemporain
}

Céline Guillot

\section{- To cite this version:}

Céline Guillot. Démonstratif et déixis discursive: analyse comparée d'un corpus écrit de français médiéval et d'un corpus oral de français contemporain. Langue française, 2006, 152, pp.56-69. halshs00324179

\section{HAL Id: halshs-00324179 \\ https://shs.hal.science/halshs-00324179}

Submitted on 22 Oct 2014

HAL is a multi-disciplinary open access archive for the deposit and dissemination of scientific research documents, whether they are published or not. The documents may come from teaching and research institutions in France or abroad, or from public or private research centers.
L'archive ouverte pluridisciplinaire HAL, est destinée au dépôt et à la diffusion de documents scientifiques de niveau recherche, publiés ou non, émanant des établissements d'enseignement et de recherche français ou étrangers, des laboratoires publics ou privés. 


\title{
Démonstratif et déixis discursive : analyse comparée \\ d'un corpus écrit de français médiéval \\ et d'un corpus oral de français contemporain
}

\author{
Céline Guillot (ENS-LSH et UMR ICAR)
}

\section{Introduction}

Notre article $^{1}$ est le fruit d'une recherche qui porte sur l'emploi du démonstratif en français, dans ce qu'il est désormais convenu d'appeler la deixis du discours. Cette recherche, qui à son commencement était centrée sur le français du Moyen âge (IX ${ }^{\text {ème }}-X \mathrm{VI}^{\text {ème }}$ siècles) et menée dans une perspective diachronique, nous a conduite à nous intéresser au français oral d'aujourd'hui et aux formes qu'y prend la deixis du discours. Nous présentons donc ici quelques-uns des principaux résultats que nous apportés l'étude comparée de deux corpus, composés pour le premier de textes écrits au Moyen âge, pour le second d'interactions orales en français contemporain.

\section{Qu'est-ce que la déixis discursive?}

\subsection{Définition}

Le terme de déixis discursive, emprunté à R. Lakoff (Lakoff 1974) et repris souvent par la suite, notamment par Ch. Fillmore (Fillmore 1975) et B. Webber (Webber 1991), est également utilisé par N. Himmelmann (N. Himmelmann 1996) pour décrire l'un des quatre contextes principaux dans lesquels les langues du monde utilisent le démonstratif. C'est la définition de Himmelmann, adoptée ensuite par Diessel dans son ouvrage sur le démonstratif et la grammaticalisation (Diessel 1999), que nous utilisons ici :

"Discourse deixis is to be understood here as reference to propositions or events. » (Himmelmann 1996 : 224).

Dans son emploi déictique discursif, le démonstratif désigne des événements ou des propositions qui ont été mentionnés dans le discours précédent. L'exemple donné par Himmelmann est le suivant :

(1) ... puis il part... et c'est la fin de cette histoire, mais après... il revient vers le paysan...

\footnotetext{
${ }^{1}$ Je remercie Jacques Jayez et Sophie Prévost, qui ont bien voulu relire ce texte. Les erreurs qui subsistent sont de mon fait.
} 
L'expression référentielle "cette histoire" illustre ici l'usage déictique discursif du démonstratif. Le déictique de discours instaure une relation anaphorique, puisqu'on a besoin des informations présentes dans le contexte qui précède l'occurrence de "cette histoire" pour interpréter l'expression référentielle, mais cette relation anaphorique est d'un type particulier. Car avec le déictique de discours on ne réfère pas par une relation de coréférence à une entité qui serait déjà donnée dans le discours, un personnage par exemple. On désigne plutôt le contenu discursif d'un ensemble de propositions qui viennent d'être énoncées, sans que ce contenu ait jamais été désigné auparavant au moyen d'une expression référentielle particulière. L'exemple (2), tiré de notre corpus de français oral, nous servira de seconde illustration :

(2)

(00:03:00)

C Doudou tu sais le p'tit (.) le mec de Doudoune (.) 'fn ça parâ̂t évident (1.5) ah/ (0.7) pendant qu'j'y [pense/...(Conversations familières) ${ }^{2}$

Dans cet énoncé, le pronom neutre ça ne désigne pas l'entité référentielle le mec de Doudoune mais plutôt le fait que Doudou soit le mec de Doudoune. Le pronom désigne donc une proposition.

Outre le fait que le déictique discursif permet d'introduire un nouveau référent dans le discours, Himmelmann constate que ce référent n'est généralement pas persistant. Il ne fait pas l'objet de reprises anaphoriques subséquentes et disparaît aussitôt du discours. Enfin, d'après N. Himmelmann toujours, la déixis du discours semble constituer le contexte d'usage typique du pronom démonstratif dans la majorité des langues. Il s'agit en effet du contexte dans lequel la fréquence du pronom est supérieure à celle des autres formes du démonstratif du déterminant démonstratif en particulier - et on constate par ailleurs que le pronom démonstratif est plus souvent déictique discursif que déictique situationnel ou anaphorique textuel (Himmelmann $1996: 225)^{3}$. On verra dans la suite de cet article que cette propriété se vérifie à toutes les époques du français, et en particulier dans le français oral d'aujourd'hui.

\subsection{Le problème de l'identification du référent}

Selon N. Himmelmann, la question de la nature du référent désigné par le démonstratif, de même que la façon dont ce référent est identifié, sont encore sujettes à discussion. La difficulté vient de ce que la taille du contexte qui sert de source à ce type d'anaphore peut varier. Ce contexte peut aller d'une simple proposition à un récit dans sa totalité. Le problème d'identification de l'objet désigné par le déictique de discours est lié au fait qu'il n'y a pas dans le contexte discursif qui précède l'occurrence du démonstratif de référent déjà donné vers lequel ce démonstratif pointerait. La seule donnée assurée dont nous disposons, c'est que le contexte-source doit être immédiatement adjacent, ou contigu, à la forme même du démonstratif. Cette exigence de contiguïté est intimement liée au caractère déictique du

\footnotetext{
${ }^{2}$ Les transcriptions du corpus de français oral sont restituées ici dans la forme qui leur a été donnée par les responsables de chaque sous-corpus. Le système de notation est organisé en tours de parole, avec changement de ligne à chaque nouveau tour de parole et identification du locuteur en début de ligne (ici C). Les pauses inférieures à une seconde sont notées (.) quand elles ne sont pas chronométrées, celles qui sont plus longues sont toujours chronométrées ((1.5) dans l'extrait). Les chevauchements sont indiqués par un crochet [ dans le tour interrompant et dans le tour interrompeur. Le signe / indique la perception d'une légère montée de la voix. Pour une description complète des conventions de transcription utilisées, voir l'url : http://clapi.univ-lyon2.fr/.

${ }^{3}$ Pour le français, on doit cependant distinguer les pronoms neutres $c e$ et ça, qui sont le plus souvent utilisés dans le contexte de la déixis du discours, et les pronoms celui-ci et celui-là qui sont généralement anaphoriques textuels.
} 
démonstratif dans cet emploi : le démonstratif désigne de façon déictique le contenu discursif d'une portion du texte qui se trouve dans son entourage immédiat.

Dans un article de 1991, B. Webber avait déjà abordé et précisé ce point grâce à son étude des pronoms that et this en anglais. Son analyse montre notamment que dans l'usage déictique discursif de ces deux pronoms démonstratifs, il est utile de distinguer le demonstratum, i. e. le contexte vers lequel pointe chaque occurrence du démonstratif, et d'autre part le référent, i. e. l'entité à laquelle le pronom réfère à chaque fois. Le passage du contexte-source de l'anaphore à l'entité à laquelle cette anaphore réfère est opéré par une relation, que Webber appelle fonction référentielle (Nunberg 1978) et dont elle dit assez peu de choses dans cette étude. On sait seulement que le contexte-source contient un certain nombre d'entités discursives qui permettent l'identification du référent désigné par le déictique de discours.

B. Webber s'intéresse davantage à la façon dont le récepteur identifie le demonstratum, ou le contexte-source, et elle utilise pour cela la notion de modèle discursif. Selon elle, la structuration du discours en segments de discours, en unités de niveaux inférieur et supérieur, doit permettre d'identifier le contexte désigné par le déictique de discours. Ce contexte-source se trouve nécessairement dans le focus du modèle de discours, ce qui, d'une certaine façon, revient à dire que ce contexte est contigu (ce qui le rend saillant) au segment dans lequel se trouve le démonstratif.

Sans aller plus avant dans l'article de B. Webber, nous souhaitons insister ici sur les problèmes que son étude soulève et auxquels elle ne répond, à notre sens, que partiellement ou pas du tout. D'une part, Webber ne donne que peu d'indications sur la façon dont on identifie le référent à partir du contexte-source. D'autre part, il faudrait définir avec plus de précision la nature des unités qu'elle appelle segments de discours et qui structurent le discours. Doit-on prendre comme unité la phrase syntaxique, la proposition, autre chose encore ? Rien n'est dit non plus de la façon dont on délimite à gauche ce contexte-source. Comment sait-on jusqu'où remonter? Quels sont les éléments qui permettent de regrouper les segments de discours dans des unités de niveau supérieur, ces unités supérieures servant de demonstratum au démonstratif ?

Notre objectif ne sera pas de répondre ici à toutes ces questions. Nous espérons toutefois donner quelques indications qui aideront peut-être à résoudre certaines d'entre elles. Nous commencerons par quelques remarques concernant les emplois déictiques discursifs du démonstratif dans un corpus médiéval.

\section{Démonstratif et déixis discursive dans un corpus médiéval}

\subsection{Fréquence des emplois déictiques discursifs du démonstratif dans le corpus médiéval}

D'après les relevés que nous avons effectués sur quatre textes narratifs en prose datés de 1400 à 1550 environ $^{4}$ (Guillot à par.), les emplois déictiques discursifs du démonstratif couvrent, selon les textes et les périodes, de $35 \%$ à $72 \%$ des occurrences de tous les démonstratifs. De même, une étude récente a montré que cet usage du démonstratif était très fréquent dans un texte en prose du $13^{\text {ème }}$ siècle, la Queste del Saint Graal (Guillot 2004). Ch. Marchello-Nizia

\footnotetext{
${ }^{4}$ Ces relevés ont été effectués grâce à la Base de Français Médiéval de l'UMR 5191 ICAR et grâce au Dictionnaire de Moyen Français de l'UMR 7118 ATILF de Nancy.
} 
(Marchello-Nizia 2006) a montré de son côté que cet emploi des déictiques se rencontre dès les plus anciens textes (dès la Passion de Clermont, mi $11^{\text {ème }}$ siècle) et qu'il a joué un rôle clé dans l'évolution sémantique générale du système déictique entre le très ancien français et l'ancien français 'classique' (voir l'article publié dans ce numéro). L'analyse détaillée d'un corpus relativement important de textes d'ancien et de moyen français montre donc qu'il s'agit là d'un des emplois majeurs du démonstratif au Moyen âge.

\subsection{Identification du référent dans le corpus médiéval}

Par ailleurs, dans tous ces textes d'ancien ou de moyen français nous avons observé une fréquence remarquable du pronom neutre $c e$, ce qui confirme les données de N. Himmelmann, mais aussi des syntagmes nominaux ceste chose, ceste aventure et ceste parole. Ces expressions référentielles ont un contenu sémantique assez abstrait (Asher 1993) et servent donc, comme le pronom neutre, à désigner de façon synthétique des contenus propositionnels qui peuvent être très variés. De ce fait aussi, il est souvent difficile d'identifier avec précision les limites du contexte qui sert de source à l'anaphore.

Dans les autres cas, nous avons tenté d'identifier les liens qui permettent de mettre en relation certaines des entités présentes dans le contexte-source avec le référent auquel réfère le déictique de discours. Dans certains énoncés, comme dans l'exemple (3), il est possible d'établir une relation morphologique et sémantique entre le nom déterminé par le déictique de discours et l'un des éléments présents dans le contexte qui le précède. Parfois, comme dans l'exemple (4), ce lien n'est que sémantique (nous avons indiqué en gras les éléments que nous mettons en relation) :

(3) En effet, ceulx là furent rompus à platte cousture et chasséz jusques au charroy, et la pluspart fuyrent jusques en la forest [...]. Les principaulx de ceste chasse estoient ces nobles de Daulphiné [...]. (Mémoires de Commynes : 28, BFM 2005)

(4) [...] il par la merveilleuse force et prouesse de soy et de ses gens desconfisi les Germanois et prinst Theutobocus leur tres orgueilleux roy, homme de grant estature [...]. Aprés ceste victoire, Marius tourna sa bataille contre les Cimbrois [...]. (Des cas des nobles hommes)

On peut supposer que dans ces énoncés la présence d'une relation sémantique (et parfois aussi morphologique) préétablie entre un ou plusieurs élément(s) du contexte-source et le nom déterminé par le déictique de discours peut guider l'identification du référent désigné par le syntagme nominal. Mais dans d'autres énoncés - et ces occurrences sont de plus en plus fréquentes avec le temps et constituent une part croissante des emplois déictiques discursifs du démonstratif - on ne peut établir aucun lien prédéfini entre l'expression anaphorique et les éléments présents dans le contexte précédent. C'est le cas notamment dans cet extrait des Mémoires de Commynes :

(5) Comme il se trouva grant et roy couronné, d'entrée ne pensa que aux vengeances, mais tost luy en vint le dommaige, et quant et quant la repentance ; et repara ceste follie et cest erreur en regaignant ceulx à qui il tenoit tort, comme vous entendrez cy après. (Mémoires de Commynes : 69, BFM 2005)

Rien ne nous dit avant l'occurrence de ceste follie et de cest erreur que le comportement du personnage qui vient d'être décrit sera qualifié de "follie" ou d'erreur". L'expression anaphorique apporte donc des informations nouvelles (comme dans les exemples traités par C. Schnedecker ici-même) et elle opère une reclassification forte sur le contenu du contexte- 
source. Il faudrait donc déterminer de quelle façon s'opère l'identification du référent introduit par le déictique de discours dans ce type d'énoncé.

Plus généralement et sur un plan macro-structurel cette fois, nous avons observé l'importance de la fonction structurante et textuelle du démonstratif déictique discursif dans le corpus de français médiéval. Toutes les anaphores indirectes que nous venons d'évoquer, appelées parfois «anaphores résomptives » (Maillard 1974), permettent en effet de subsumer le contenu du discours précédent grâce au pronom démonstratif ou grâce à la tête lexicale du SN démonstratif, le substantif choisi permettant de sous-catégoriser et de résumer le contenu du contexte-source (Marandin 1986, Fraurud 1992, Francis 1994, Botley 2006).

En outre, dans la plupart de ces occurrences le déictique discursif est employé dans des contextes de rupture ou de transition textuelle. L'expression aprés ceste parole, par exemple, est le plus souvent placée à la frontière entre une séquence au discours direct et une séquence narrative. Elle marque ainsi la clôture de la première et l'ouverture de la seconde. De la même façon, lorsqu'il est utilisé à l'intérieur du récit narratif, le déictique de discours indique le plus souvent une frontière discursive entre deux séquences narratives. Dans les manuscrits médiévaux, dont le mode d'organisation textuelle et matérielle diffère de celui des imprimés modernes, le déictique discursif joue le rôle d'un marqueur de structuration dont la fonction se compare à celle d'un signe de ponctuation (l'alinéa de paragraphe par exemple, ou les guillemets de fin du discours direct) dans l'imprimé moderne.

C'est à la lumière de cette première analyse et de ces premiers résultats que nous avons abordé notre corpus de français oral contemporain.

\section{Démonstratif et déixis discursive dans un corpus de français oral contemporain}

\subsection{Remarques générales}

Notre corpus oral est composé de quatre sous-corpus distincts ${ }^{5}$. Il s'agit de quatre interactions de sept minutes chacune, ce qui est peu, mais on verra que l'analyse de cet ensemble limité soulève à elle seule un assez grand nombre de problèmes, qui sont parfois difficiles à résoudre. Ces sous-corpus sont les suivants :

- conversations familières, extrait clodif q4 (Véronique Traverso)

- UCPB, extrait Ginette (François Lupu)

- conférence ordinaire, extrait (Catherine Orecchioni)

- retouches-papotages, extrait enregistrement 1 - agrafe (Liliane Vosghanian)

Ces quatre sous-corpus forment un ensemble assez hétérogène. D'une part, ils sont très différents du point de vue générique : $U C P B$, extrait Ginette, est une interaction médicale qui concerne un médecin et sa patiente ${ }^{6}$; conférence ordinaire est une interaction didactique (il s'agit du début d'une communication enregistrée lors d'un colloque); conversations familières est une interaction privée enregistrée dans un appartement; retouches-papotages est une interaction à la fois privée et de commerce (des habitués viennent dans un magasin de retouche pour effectuer des transactions commerciales et aussi pour discuter avec la

\footnotetext{
${ }^{5}$ Ces différents sous-corpus appartiennent tous à la Base CLAPI (Corpus de Langues Parlées en Interaction), élaborée et gérée par l'UMR 5191 ICAR.

${ }^{6}$ Nous utilisons dans cette étude la classification générique qui organise la base CLAPI.
} 
responsable du magasin). Le nombre de participants varie également d'un sous-corpus à l'autre: l'interaction didactique est un monologue, l'interaction médicale et retouchespapotages sont des dilogues et conversations familières est un quadrilogue. Enfin et surtout, on suppose une différence de registre très nette entre l'interaction didactique, plus "soutenue" ou plus "formelle", et toutes les autres interactions, les interactions privées surtout.

Dans trois des quatre sous-corpus que nous avons choisis, le pronom neutre ça a une fréquence tout à fait remarquable. Le monologue didactique seul fait exception, et ce fait est sans aucun doute à mettre en relation avec les caractéristiques typologiques des différents sous-corpus que nous venons de mentionner. On a constaté à plusieurs reprises combien la fréquence de ça pouvait varier selon les types de données analysées et les registres de langue utilisés (Maillard 1989). Il apparaît donc que ça est peu employé dans les situations d'échange les plus formelles, comme le sont par exemple les communications présentées lors de rencontres scientifiques ${ }^{7}$.

Dans les trois autres sous-corpus, la fréquence de ça est tout à fait frappante si on la compare à celle de toutes les autres lexies. Dans les conversations familières, ça est même le mot le plus fréquent. Cette fréquence est également remarquable si on la compare aux occurrences de tous les démonstratifs dans chacun des trois sous-corpus. Le tableau suivant, qui donne ces fréquences en valeur absolue et en valeur relative ${ }^{8}$, le montre très clairement :

Tableau 1 : Fréquence de "ça" par rapport aux autres formes du démonstratif

\begin{tabular}{|l|l|l|}
\hline corpus & fréquence des démonstratifs & fréquence de ça \\
\hline conversations familières & 99 & $44(44,4 \%)$ \\
\hline UCPB & 66 & $20(30,3 \%)$ \\
\hline conférence ordinaire & 57 & $6(10,5 \%)$ \\
\hline retouches-papotages & 138 & $53(38,4 \%)$ \\
\hline
\end{tabular}

Compte-tenu de ces données, nous avons choisi de nous centrer sur les occurrences de ça dans nos interactions orales, ce qui explique également que dans la suite de cet article il ne sera pour ainsi dire plus question du sous-corpus conférence ordinaire, dans lequel ça est très peu représenté.

\section{2. Ça est-il toujours déictique discursif ?}

La déixis du discours constituant, d'après Himmelmann, le contexte d'emploi privilégié du pronom démonstratif, on peut légitimement se demander si ça est toujours déictique discursif dans le corpus que nous analysons. L'examen du corpus montre qu'en réalité les exemples dans lesquels ça est un déictique situationnel ou un pronom de reprise anaphorique ne manquent pas. L'énoncé (6) illustre ces deux types d'emploi. Le premier ça est un déictique situationnel, le second un pronom de reprise :

(6)

$(00: 00: 00)$

Mme $N$ - et puis j' mords dedans comment chacun fait hein (.) dites y'avait une agrafe j'l'ai pas apportée je l'ai oubliée j'vous l'apporterai (.) comme ça dans le pain $=$ (montre la taille de l'agrafe avec les mains $)$

$P-\quad=$ dans le pain $\uparrow$

\footnotetext{
7 Il semble plus difficile d'établir un lien entre le peu d'occurrences de ça et le caractère monologal caractéristique de cette interaction.

${ }^{8}$ Les chiffres décrivent la fréquence de ça en nombre absolu d'occurrences et relativement au nombre d'occurrences total des démonstratifs dans chaque sous-corpus (pourcentages).
} 


\section{Mme N-ça s'est accroché [dans mon palais (retouches-papotages) ${ }^{9}$}

Il arrive fréquemment aussi que ça soit non référentiel et qu'il entre dans des tournures impersonnelles du type de celle qu'on trouve en (7) :

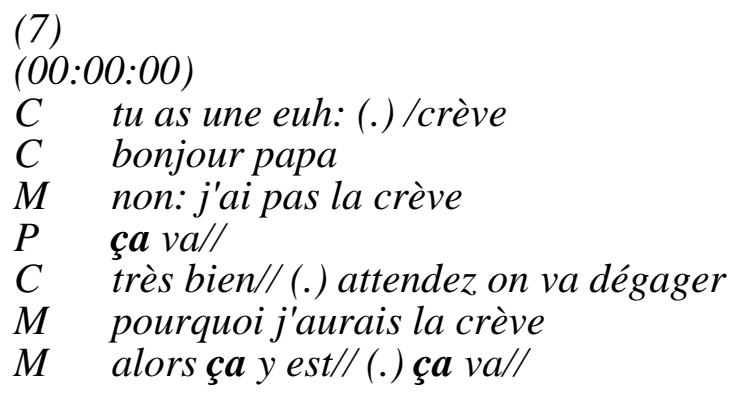

Néanmoins, le tableau suivant montre que dans deux des trois sous-corpus analysés les occurrences du ça déictique de discours représentent environ la moitié des occurrences du pronom neutre :

Tableau 2 : Fréquence des emplois déictiques discursifs de ça

\begin{tabular}{|l|l|l|}
\hline corpus & fréquence de $c ̧ a$ & fréquence de ça déictique discursif \\
\hline conversations familières & 45 & $10 / 22,2 \%$ \\
\hline UCPB & 20 & $10 / 50 \%$ \\
\hline conférence ordinaire & 6 & 3 \\
\hline retouches-papotages & 53 & $25 / 47,2 \%$ \\
\hline
\end{tabular}

On a montré déjà (Maillard 1989, Kleiber 1987, Godart-Wendling 2000) que le pronom neutre ça permet, comme le substantif chose, de désigner des objets qui ne sont pas nommés ou qui ne sont pas classifiés et qu'il désigne le plus souvent les objets discursifs sans nom que sont les procès ou les événements. On ne saurait donc s'étonner que ça soit fréquemment utilisé comme un déictique de discours dans le corpus.

\subsection{Comment identifier le référent qui est désigné par ça ?}

Dans la mesure où, comme on vient de le voir, le pronom ça permet de désigner un objet qui n'est pas nommé, il est souvent difficile d'identifier précisément l'entité à laquelle il réfère. On peut même se demander si en utilisant ça le locuteur ne veut pas au contraire éviter de référer à une entité particulière. Dans ce cas, plus qu'à désigner un objet précis, ça servirait avant tout à référer, de façon déictique, à ce qui vient d'être dit avant sa propre occurrence dans le discours. D'où l'intérêt qu'il y a pour nous à décrire le plus finement possible la façon dont est délimitée la portion du discours qui sert de source au ça déictique discursif.

Ainsi s'explique sans doute aussi l'apparente transparence référentielle de ça. Si ça ne réfère à aucune entité référentielle précise mais à une sorte d'objet discursif qui n'a pas de nom, on comprend dès lors que les locuteurs s'accommodent très bien d'une série d'occurrences qui

\footnotetext{
${ }^{9}$ Le signe = indique un enchaînement immédiat entre deux tours, et il apparaît à la fin du premier tour et au début du suivant. Le non-verbal est noté entre parenthèses. Le crochet [ indique un chevauchement. Le signe / indique la perception d'une légère montée de la voix. Le signe $\uparrow$ indique la perception d'une forte montée de la voix.
} 
posent au contraire toutes sortes de problèmes quand on tente d'identifier précisément un référent de discours auquel il pourrait référer.

C'est dans la perspective ouverte par ces quelques remarques préalables que nous avons analysé les différents emplois de ça déictique discursif dans notre corpus de français oral. Cette analyse nous a permis d'identifier trois types de contexte-source :

- le contexte-source est constitué des dernières paroles de l'interlocuteur

- le contexte-source est constitué des dernières paroles du locuteur lui-même

- le contexte-source est constitué de l'ensemble du discours qui précède l'occurrence de ça

\subsection{1. Ça réfère au contenu des dernières paroles de l'interlocuteur}

Il est assez fréquent qu'en utilisant ça le locuteur réfère au contenu des paroles de l'interlocuteur dont le tour de parole vient de se terminer :

(8)

(00:01:00)

Mme $N-=$ mais c'est dans mais c'est sorti [de mon morceau

$P$ - $\quad$ [oui c'est dans le pain ça $=$

Mme $N$ - = mais c'est dans l' pain

$P$ - $\quad$ fff (P souffle) c'est incroyable [ça...(retouches-papotages)

On voit que, dans ce type d'énoncé, le contexte-source est nettement distinct du segment de discours dans lequel se trouve l'occurrence de ça, segment que nous appellerons désormais le contexte hôte (que F. Cornish 1999 appelle le «segment indexical »). A la rupture syntaxique s'ajoute la rupture qui est créée par le changement de locuteur. Le contexte-source et le contexte hôte étant nettement disjoints, l'identification des deux s'en trouve facilitée.

\subsection{2. Ça réfère au contenu des dernières paroles du locuteur lui-même}

Le cas le plus fréquent est celui dans lequel le locuteur désigne par ça le contenu des paroles qu'il vient de prononcer dans un énoncé qui est syntaxiquement disjoint de celui dans lequel se trouve le pronom neutre. A la rupture syntaxique s'ajoute généralement la présence d'autres indices, qui indiquent eux aussi qu'on change d'unité discursive. La pause, marquée dans l'exemple (9) par le point entouré de parenthèses (.), fait partie de ces indices. Elle renforce la structure syntaxique de l'énoncé et joue ici un rôle séparateur et organisateur (Lacheret 1999 : 52) :

(9)

(00:02:00)

M j'ai pris la-la rue qu'i m'a montré (.) ça nous f'sait gagner du [temps hein... (conversations familières) $^{10}$

Sont également souvent placés à la frontière entre le contexte-source et le contexte hôte des mots du discours ou marqueurs discursifs du type de hein, fin, bon fin, qui peuvent servir de «ponctuants » du discours (Vincent 1993). Ces marqueurs jouent parfois dans la structuration discursive un rôle qui est assez comparable à celui de la pause, et ils se combinent d'ailleurs souvent avec elle :

(10)

\footnotetext{
${ }^{10}$ Le tiret indique un mot interrompu brutalement par le locuteur.
} 
(00:04:00)

$P$ - $\quad$ [ah $i$ - sont vraiment non non mais là là ce ce qui a fait bah il avait vraiment une bonne intention de faire du mal hein ça ç'est c'est
évident... (retouches-papotages)

Il apparaît donc que lorsque ça réfère au contenu propositionnel des dernières paroles du locuteur, un ensemble d'indices (syntaxiques, suprasegmentaux, mots du discours) contribuent à disjoindre nettement le contexte-source du contexte hôte. Ces différents marqueurs indiquent qu'on passe d'une unité à la suivante, et ça désigne l'énoncé (qu'il faudrait délimiter à gauche avec davantage de précision) qui précède immédiatement la rupture.

Le second cas de figure, nettement moins fréquent que le premier, est celui dans lequel ça joue le rôle d'un pronom de reprise après une dislocation à gauche. Nous nous intéressons ici uniquement aux énoncés dans lesquels l'élément détaché à gauche n'est pas une entité référentielle mais une proposition. L'exemple (11) nous servira d'illustration :

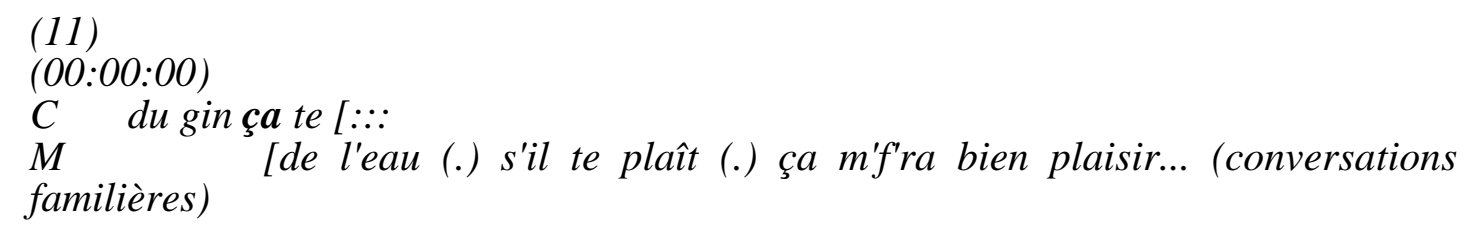

Dans cet énoncé, nous considérons que le pronom ça ne désigne pas l'entité référentielle le gin, mais plutôt une proposition du type «boire du gin » ou bien «si je te donne du gin » ou bien autre chose d'approchant. D'un point de vue syntaxique, dans ce type d'énoncé aussi le contexte-source et le contexte hôte sont nettement disjoints, l'élément détaché qui sert de source au déictique discursif étant extraposé à la proposition syntaxique dans lequelle il se trouve.

Ce type d'exemple peut être rapproché d'énoncés dans lesquels le pronom ça anaphorise une première occurrence de ça, utilisé comme déictique de discours. L'énoncé (12) en offre un bon exemple :

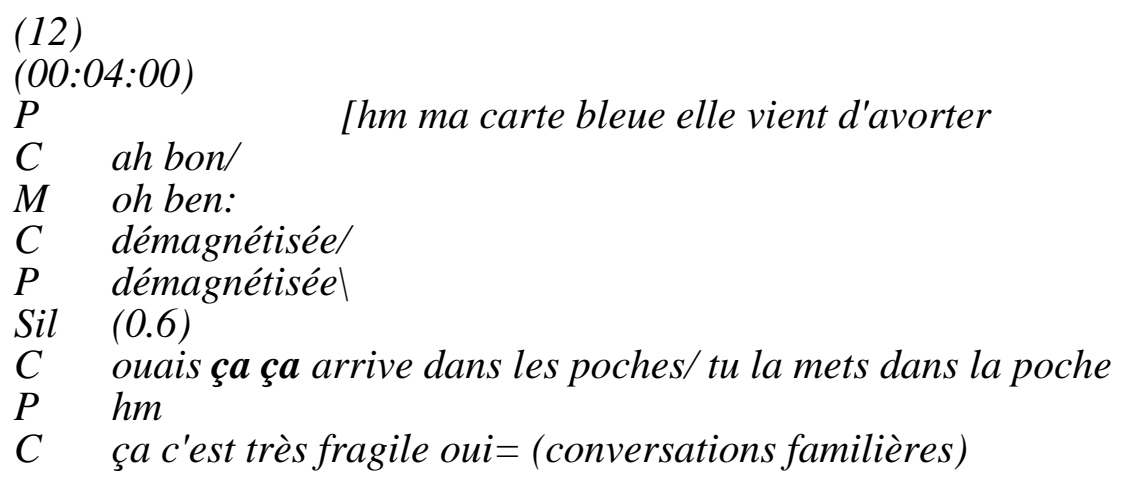

\subsection{3. Ça réfère au contenu de l'ensemble du discours qui précède son occurrence}

Enfin, il est aussi quelques énoncés, comme (13), dans lesquels le pronom neutre ne semble pas référer au contenu du contexte immédiat de son occurrence mais au contenu de l'ensemble du discours qui le précède :

\footnotetext{
${ }^{11}$ Il est question dans ce passage des personnes qui, entre autres méfaits, ont emporté le paillasson de la cliente du magasin de retouche.
} 
(13)

(00:03:00)

Mme $N$

[puis je je savais pas avec quoi avec quoi me me me désinfecter

la bouche quoi alors j'ai pensé à l'Eludril tout bonnement c'est tout ce que j'avais (.) pouvais quand même pas prendre de l'eau d' javel hein (rires de L)

Mme $N \quad$ alors bon ben voilà (.) à part ça ça va /

On interprète la première occurrence de ça comme référant au contenu de toute la discussion qui s'est tenue jusque là. De même, en (14) on peut considérer que ça pointe de façon déictique vers tout le segment de discours qui précède et qu'il réfère au contenu de l'ensemble de ce discours :

(14)

$(00: 06: 07)$

Mme $N$-... bon et ben voilà c'est en mangeant qu'j'ai trouvé c'est même j'aurai pu en en coupant mon machin en deux [la trouver ben non c'était pas dit quoi c'est dit qu'j'aurai des pépins (.)

$P-\quad$ [bah oui

Mme $N$ - 'fin c'est pas marrant mais y'a pire que ça mais j'me suis dit un enfant [moi tout ... (retouches-papotages)

Mais on peut considérer aussi que dans ces exemples ça ne réfère pas à proprement parler au contenu propositionnel mais plutôt au topique du discours qui précède. Dans l'exemple (13), la partie de l'énoncé dans laquelle se trouve la première occurrence de ça annonce une transition discursive. Enoncer à part ça revient à indiquer qu'on va changer de sujet de discussion, que le thème de discours en cours va laisser place au suivant. L'exemple (14) se laisse analyser de la même façon. Au centre de l'interlocution se trouve le récit fait par l'une des clientes du magasin de retouche de ce qui lui est arrivé le matin même : elle s'est planté une agrafe dans le palais en mordant dans sa tartine de pain. Ce récit, assez extraordinaire en soi, revient continuellement dans la discussion entre les deux protagonistes et constitue le topique de discours qui domine la plus grande partie de l'interlocution.

Le fait que ça puisse parfois référer au thème de discours dominant nous amène naturellement à aborder la fonction pragmatique et discursive de ça dans le corpus de français oral.

\subsection{Fonction pragmatique et discursive de ça}

Comme c'était le cas déjà dans les textes français écrits au Moyen âge, il semble que le démonstratif déictique, et en particulier le pronom neutre ça, joue un rôle tout à fait essentiel dans l'organisation et la structuration du discours oral.

D'un point de vue pragmatique, on constate que ça désigne toujours une information connue et saillante, déjà donnée dans le discours qui précède son occurrence (Prince 1981). Ça a le plus souvent aussi un rôle topical : il a avec le reste de l'énoncé une relation d'à propos («aboutness ») et il est fréquemment - mais pas toujours - le sujet syntaxique de la proposition dans laquelle il se trouve. En outre, ça est lui-même souvent topicalisé, soit dans une dislocation à gauche (exemple 15), soit dans une dislocation à droite (exemple 16) :

(15)

(00:03:00) 
et ça ça a commencé après la ménopause... (UCPB) ${ }^{12}$

(16)

$(00: 01: 00)$

Mme $N$ - = mais c'est dans mais c'est sorti [de mon morceau

$P$ - $\quad$ [oui c'est dans le pain ça $=$

Mme $N$ - = mais c'est dans l'pain

$P-\quad f f f\left(P\right.$ souffle) $c^{\prime}$ 'est incroyable [ça...(retouches-papotages)

Qu'un élément dont le contenu sémantique est relativement abstrait puisse ainsi être topicalisé dans l'énoncé a, à première vue, de quoi surprendre. Si l'on considère, comme le fait $\mathrm{K}$. Lambrecht (2001), qu'un élément disloqué à gauche sert à instaurer une relation topicale nouvelle à l'intérieur du discours, l'élément disloqué étant lui-même accessible ou inférable, il est légitime de se demander comment le pronom neutre ça, qui ne dénomme aucune entité référentielle concrète, peut introduire ce type de relation.

La réponse se trouve, à notre sens, dans le type particulier de relation qu'instaure ça. S'il ne sert pas à désigner un objet de discours précis, à propos duquel il va être énoncé quelque chose dans la suite de l'énoncé - ce qui est normalement le cas d'un topique de phrase - c'est sans doute parce qu'en vertu de sa valeur sémantique ça a souvent la fonction pragmatique d'un opérateur de topicalisation. Sa fonction pragmatique consiste alors à faire du discours qui précède le topique du discours qui suit ${ }^{13}$, et il n'a donc pas à dénommer un nouveau topique de discours ni à introduire un topique de phrase.

\section{Conclusion}

Notre étude comparative, menée à partir de données de langue ancienne et de langue orale sur le démonstratif déictique de discours, et en particulier sur le pronom neutre ça dans le français oral d'aujourd'hui, confirme que le déictique de discours est interprété relativement au contenu discursif d'une partie du contexte qui précède sa propre occurrence, ce contexte discursif étant à la fois nécessairement contigu et en même temps nettement disjoint de l'énoncé dans lequel se trouve le démonstratif. Il est clair aussi que la limite gauche de ce contexte peut varier, puisqu'il va d'une simple proposition à l'ensemble du discours qui précède et qui a été produit jusqu'à l'occurrence du déictique lui-même. La question des critères qui permettent de délimiter la borne gauche de ce contexte reste ouverte.

Mais le point qui ressort le plus clairement de notre étude concerne le rôle que le déictique de discours joue dans l'organisation discursive, et, pour ce qui concerne l'oral, dans la gestion progressive du topique de discours. Dans le cas de dialogues où la progression topicale se construit dans l'interaction entre les différents participants et où l'on passe beaucoup de temps à reprendre ce qu'a dit l'interlocuteur pour en dire autre chose, il n'est pas surprenant que ça, déictique de discours le plus fréquent à l'oral, joue un rôle discursif et pragmatique de premier plan et serve ainsi à faire avancer le dialogue du point de vue informationnel.

\footnotetext{
${ }^{12}$ Le médecin interroge ici sa patiente et tente de trouver l'origine de ses ronflements nocturnes. Toute l'interaction tourne autour de cette question.

${ }^{13}$ Pour les distinctions entre topique de phrase, topique et thème de discours, voir notamment Prévost 1998.
} 


\section{$\underline{\text { Références }}$}

Apothéloz, D. (1995). Rôle et fonctionnement de l'anaphore dans la dynamique textuelle, Genève-Paris, Droz.

Asher, N. (1993). Abstract Objects in Discourse, Dordrecht, Kluwer.

Botley, S. P. (2006). «Indirect anaphora », International Journal of Corpus Linguistics, 11/1, p. 73-112.

Cornish (1999), F. Anaphora, Discourse and Understanding, Oxford, Clarendon Press.

Diessel, H. (1999). Demonstratives. Form, Function, and Grammaticalization, AmsterdamPhiladelphia, John Benjamins Publishing Company.

Fillmore, C. (1975). Santa Cruz Lectures on Deixis, Bloomington, Indiana University Press.

Francis, G. (1994). «Labelling discourse: an aspect of nominal-group lexical cohesion », M. Coulthard (éd.), Advances in Written Text Analysis, London, Routledge, p. 83-101.

Fraurud, K. (1992). Processing Noun Phrases in Natural Discourse, Thèse de doctorat, Université de Stockholm.

Godart-Wendling, B. (2000). «Comment ça réfère ? », Revue de Sémantique et Pragmatique, 7, p. 105-121.

Grenoble, L. (1994). «Discourse deixis and information tracking », Berkeley Linguistics Society, 20, p. 208-219.

Guillot, C. (2004). «Ceste parole et ceste aventure dans la Queste del Saint Graal, marques de structuration discursive et transitions narratives », L'Information grammaticale, 103, p. 29-36.

Guillot, C. (à par. 2006). «Entre anaphore et deixis : l'anaphore démonstrative à fonction résomptive », à paraître aux éditions Niemeyer dans les Actes du XXIV ${ }^{\text {ème }}$ Congrès de Linguistique et de Philologie Romanes organisé à Aberystwyth (août 2004)

Himmelmann, N. (1996) : «Demonstratives in narrative discourse : a taxonomy of universal uses », B. Fox (éd.), Studies in Anaphora, Amsterdam/Philadelphia, John Benjamins Publishing Company, p. 205-254.

Kleiber, G. (1984). «Sur la sémantique des descriptions démonstratives », Linguisticae Investigationes, 8, p. 63-85.

Kleiber, G. (1987). « Mais à quoi sert donc le mot chose? Une situation paradoxale », Langue française, 73, p. 109-128.

Lacheret, A. (1999). La prosodie du français, Paris, Editions du CNRS.

Lakoff, R. (1974). "Remarks on this and that», Proceedings of the Chicago Linguistics Society, Chicago, p. 345-356.

Lambrecht, K. (2001). «Dislocation », M. Haspelmath et al. (éd.), Language Typology and Language Universals, Berlin/New York, Mouton de Gruyter, p. 1050-1078.

Maillard, M. (1974): «Essai de typologie des substituts diaphoriques (Supports d'une anaphore et/ou d'une cataphore) », Langue française, 21, p. 55-71.

Maillard, M. (1989). Comment "ça" fonctionne (ou Etude du fonctionnement de "ça" en français moderne dans la perspective d'une linguistique génétique), Thèse d'Etat, Université de Paris 10.

Marandin, J.-M. (1986). «Ce est un autre. L'interprétation anaphorique du syntagme démonstratif », Langages, 81, p. 75-90.

Marchello-Nizia, C. (2006). «From personal deixis to spatial deixis : The semantic evolution of demonstratives from Latin to French », M. Hickman et M. St Robert (éd.), Space in languages, linguistic systems and cognitive categories, Amsterdam, John Benjamins Publishing Company, chapitre 5.

Nunberg, G. (1978). The Pragmatics of Reference, Bloomington, IULC.

Prévost, Sophie (1998). «La notion de thème : flou terminologique et conceptuel », Cahiers de praxématique, 30 , p. 13-35. 
Prince, E. (1981). « Toward a Taxonomy of Given-New Information ”, P. Cole (éd.), Radical Pragmatics, New York, Academic Press, p. 223-255.

Schmid, H.-J. (2000). English Abstract Nouns as Conceptual Shells, Berlin/New York, Mouton de Gruyter.

Vincent, D. (1993). Les ponctuants de la langue et autres formes du discours, Québec, Nuit Blanche éditeur.

Webber, B. L. (1991). «Structure and Ostension in the Interpretation of Discourse Deixis », Language and Cognitive Processes, 6 (2), p. 107-135.

\section{Corpus}

Ancien et moyen français

- Barbance (Guillot), (1993). Edition critique et commentaire linguistique du «Des cas des nobles hommes et femmes » (Laurent de Premierfait, 1409), Thèse de l'Ecole Nationale des Chartes.

BFM - Base de Français Médiéval [En ligne]. Lyon: UMR ICAR / ENS-LSH, 2005, $<$ http://bfm.ens-lsh.fr>.

DMF - Dictionnaire de Français Médiéval [en ligne]. Nancy : UMR 7118.

Français oral contemporain (Base CLAPI)

- conversations familières, extrait clodif $q 4$ (Véronique Traverso) : http://clapi.univ-lyon2.fr/

- UCPB, extrait Ginette (François Lupu) : http://clapi.univ-lyon2.fr/

- conférence ordinaire, extrait (Catherine Orecchioni) : http://clapi.univ-lyon2.fr/

- retouches-papotages, extrait enregistrement 1 -agrafe (Liliane Vosghanian):

http://clapi.univ-lyon2.fr/ 